\title{
La educación y la distribución del tiempo laboral como variables para la selección de personal y la eficiencia organizacional ${ }^{1}$
}

Cómo citar este artículo / To reference this article / Comment citer cet article / Para citar este artigo:

Candelo-Viáfara, J. M. (2020). La educación y la distribución del tiempo laboral como variables para la selección de personal y la eficiencia organizacional. Revista Escuela de Administración de Negocios, (88), 49-62. DOI: https://doi.org/10.21158/01208160. n88.2020.2498

\section{Resumen}

Este documento tiene como objetivo plantear un modelo analítico que permita mejorar la elección del recurso humano en las organizaciones a fin de aumentar la eficiencia organizacional. Para realizar dicho modelo se utilizan la hermenéutica, la estática comparativa y la narrativa como herramientas fundamentales de investigación; también se analizan factores importantes a tener en cuenta durante la selección de personal como la satisfacción laboral, la productividad y los salarios de eficiencia. El modelo planteado pretende aumentar la productividad y el bienestar de los colaboradores, así como reducir la incertidumbre al momento de tomar decisiones en las organizaciones; pues, teniendo en cuenta existen diversos cargos con dedicación temporal distinta, se basa en tres variables fundamentales: el tiempo extralaboral del individuo, el tiempo que el individuo está dispuesto a permanecer trabajando y el nivel de educación que tiene; siendo estas dos últimas las de mayor trascendencia y las que permiten concluir que en el caso de la alta gerencia, las personas que ocupen estos cargos deben ser individuos con un alto nivel de conocimiento, dispuestos a sacrificar mucho de su tiempo trabajando, mientras que servicios como el de vigilancia lo deben ocupar personas dispuestas a sacrificar mucho de su tiempo en el trabajo, que tengan bajos niveles de educación.

Palabras claves: recursos humanos; talento humano; selección de talento humano; selección de personal; tiempo laboral; tiempo extralaboral; eficiencia organizacional.

\footnotetext{
${ }^{1}$ Este documento se desarrolla en el marco de la tesis doctoral del autor para optar al título de Doctor en administración de la Universidad del Valle.

${ }^{2}$ Estudiante de doctorado en administración de la Universidad del Valle, Magíster en Economía Aplicada de la Universidad del Valle Colombia, Administrador de empresas de la Universidad del Valle Colombia. ORCID: https://orcid.org/0000-0002-4152-5597
} 


\title{
Education and work time distribution as variables for the selection of person-nel and organizational efficiency
}

\begin{abstract}
This document aims at proposing an analytical model that helps improve the selection of the human resource in organizations, in order to increase organizational efficiency. In the interest of carrying out such a model, we used fundamental research tools such as hermeneutics, comparative statics, and narrative. Important factors to be taken into account during personnel selection are also analyzed, such as job satisfaction, productivity, and efficiency wages. The purpose of the model that is here proposed is to increase the productivity and the welfare of employees, as well as to reduce uncertainty when making decisions in organizations; and taking into account that there are different positions with different time availability requirements, it is based on three key variables: the non-work time of the individual, the time the individual is willing to stay at work, and the level of education of the individual. The last two are the most important and allow us to conclude that, in the case of senior management, people who run for these positions should be individuals with a high level of knowledge, willing to sacrifice much of their time at work; while services such as surveillance should be occupied by people willing to sacrifice much of their time at work and who have low levels of education.
\end{abstract}

Keywords: human resources; human talent; human talent selection; personnel selection; working time; non-working time; organizational efficiency.

\section{Educação e distribuição do tempo de trabalho como variáveis para seleção de pessoal e eficiência organizacional}

\section{Resumo}

Este documento tem como objetivo propor um modelo analítico que permita melhorar a escolha de recursos humanos nas organizações, a fim de aumentar a eficiência organizacional. Para fazer esse modelo, hermenêutica, estática comparativa e narrativa são usadas como ferramentas fundamentais de pesquisa. Também são analisados fatores importantes a serem considerados durante a seleção de pessoal, como satisfação no trabalho, salários de produtividade e eficiência. O modelo proposto visa aumentar a produtividade e o bem-estar dos funcionários, além de reduzir a incerteza na tomada de decisões nas organizações; portanto, levando em consideração várias posições com dedicação temporária diferente, ela se baseia em três variáveis fundamentais: o tempo extra-trabalho do indivíduo, o tempo que o indivíduo deseja continuar trabalhando e o nível de escolaridade que possui; sendo os dois últimos os mais importantes e os que permitem concluir que, no caso da alta administração, as pessoas que ocupam esses cargos devem ser indivíduos com um alto nível de conhecimento, dispostos a sacrificar grande parte do tempo trabalhando enquanto prestam serviços. Como a vigilância, ela deve ser ocupada por pessoas dispostas a sacrificar grande parte do tempo no trabalho, com baixos níveis de educação.

Palavras-chave: recursos humanos; talento humano; seleção de talento humano; seleção de pessoal; Expediente; hora extra; eficiência organizacional. 


\section{Éducation et répartition du temps de travail: variables} de sélection du personnel et de l'efficacité organisationnelle

\section{Résumé}

Ce document propose un modèle analytique permettant d'améliorer le recrutement de ressources humaines en entrepises afin d'augmenter l'efficacité organisationnelle. Pour réaliser ce modèle nous avons utilisé l'herméneutique, la statique comparative et le récit narratif comme outils de recherche fondamentale. Les principaux facteurs à respecter lors de la sélection du personnel sont la satisfaction au travail, la productivité et l'efficiacité salariale. Le modèle proposé vise à accroître la productivité et le bien-être des employés ainsi qu'à réduire l'incertitude lors de la prise de décisions dans les organisations; et par conséquent, compte tenu du fait qu'il existe différents postes exigeants des contraintes horaires différentes, trois variables d'ajustement fondamentales sont prises en considération: le temps extraprofessionnel que l'employé est prêt à octroyer à l'entreprise, le temps que l'employé est réellement disposé à travailler et le niveau d'éducation de la personne; les deux derniers éléments étant les plus importants et ceux nous permettant de conclure qu'au niveau des directions, les personnes occupant ces postes doivent démontrer un haut niveau de connaissances, être disposées à sacrifier une grande partie de leur temps à leur profession, tandis que des emplois ayant trait à la surveillance seront occupés par des personnes peu diplomées mais également prêtes à sacrifier une grande partie de leur temps à leur travail.

Mots-clés: ressources humaines; sélection des ressources humaines; sélection du personnel; temps de travail; heures supplémentaires; efficacité organisationnelle. 


\section{Introducción}

$\mathrm{U}$ na de las premisas que plantean autores como Taylor (1914) y Fayol (referenciado por Shafritz, Ott y Jang, 2015) es: «La persona correcta en el lugar correcto». Esta afirmación abre la puerta a contemplar la importancia que tiene el recurso humano en la organización, ya que si una persona no se encuentra en el lugar adecuado puede causar grandes pérdidas de eficiencia en los procesos empresariales. De acuerdo con esto, desde los cimientos de la teoría de las relaciones humanas, Elton Mayo (1933) realizó diferentes experimentos en los que identifica cómo el factor salario y otros beneficios económicos no eran la única pieza fundamental para el aumento de la productividad. El aumento de la productividad también está relacionado con el trato y la reafirmación como ser humano que el individuo tiene en las organizaciones. De acuerdo con Maslow (1954), el ser humano tiene una serie de necesidades en su vida. Tres de ellas son: la afiliación, la estima y la autosuperación. Por consiguiente, para una mejor gestión del recurso humano y el aumento de la productividad se debe identificar dónde los individuos suplen estas necesidades: ¿en el trabajo o en el tiempo extralaboral?

La contribución de esta investigación recae en la identificación de variables relevantes que permitan seleccionar el mejor individuo para el cargo adecuado, ya que, si la persona puede satisfacer sus necesidades personales, lo más probable es que aumente su productividad (Harmermesh 1977; Herzberg, Mausner y Snyderman, 1959; Maslow, 1954). Esto permitiría que la organización se beneficie del mayor potencial de cada persona, aumente la eficiencia organizacional, mejore el bienestar y reduzca la incertidumbre organizacional. Ahora bien, aunque este documento presenta como contribución la identificación de variables para una mejor toma de decisiones en el momento de seleccionar el personal para un cargo determinado, no busca ser un modelo absolutista orientado a la selección, sino una guía que permita la mejor elección de los individuos en un cargo determinado.

Por tanto, este documento tiene como objetivo plantear un modelo analítico dirigido a mejorar la elección del recurso humano en las organizaciones con el fin de aumentar la eficiencia organizacional. Se plantean las variables de educación y preferencias del individuo por el tiempo laboral y extralaboral. Como base se tendrá la teoría de salarios de eficiencia, apoyada en el nivel de educación y las necesidades de afiliación, estima y autosuperación. Este documento se divide en cinco secciones: la introducción, una revisión de literatura, el desarrollo del modelo y, por último, las conclusiones. 


\section{Revisión de literatura}

\subsection{Selección de personal}

Henri Fayol, en cuanto es uno de los precursores de la administración contemporánea, plantea la organización desde una forma integral. Así, al desglosar la administración como eje fundamental, traza las cinco funciones que, según él, debe realizar una persona que juega un papel administrativo. Estas son: prever, organizar, mandar, coordinar y controlar. Esto en razón a que se debe prever lo que se avecina, organizar todos los recursos sociales, coordinar los factores humanos e industriales, vigilar las funciones para que todo marche de la mejor manera y decir qué hacer. Taylor (1914) y Fayol (referenciado por Shafritz, Ott y Jang, 2015) concuerdan en un punto muy relevante que asume como problemática este documento: «Se debe tener la persona correcta en el lugar correcto».

Las investigaciones contemporáneas de recursos humanos han identificado que para encontrar empleados de un alto rendimiento se deben plantear entornos laborales que permitan el crecimiento personal, retos, compromiso y oportunidades de crecimiento (Collings y Mellahi, 2009; Harter y Blacksmith, 2010). Por tanto, autores como Herriot (2002), o Morgeson y Dierdorff, (2011), identifican cómo en el propósito de atraer y retener personal de alto rendimiento organizacional se puede promover un entorno que permita integrar las expectativas de crecimiento personal del empleado y las labores organizacionales. En este sentido, la literatura del ajuste adecuado del personal identifica que la selección del recurso humano es una de las claves para la retención y el compromiso de los empleados frente a la organización, de manera que esta pueda enfrentar el entorno competitivo (Kristof, 2006). Del mismo modo, Verquer, Beehr y Wagner (2003), así como May, Gilson y Harter (2004), y Saks y Gruman (2011), señalan que la selección correcta del personal está asociada con el compromiso del empleado y el rendimiento organizacional.
Las estrategias de recursos humanos se focalizan en aumentar la competitividad y el compromiso de los trabajadores. Por tanto, en la medida en que las empresas requieran personal que esté alineado con la organización, la selección de este debe dar luces que permitan identificar los empleados potenciales con relación a este objetivo (Macey y Schneider, 2008), y así la organización esté en capacidad de reclutar el empleado más adecuado para el cargo (Guest, 2014). Autores como, por ejemplo, Vance (2006), argumentan que seleccionar el individuo más acorde a la cultura organizacional permite generar un mayor compromiso del empleado. En este sentido, características tales como la personalidad y las expectativas pueden influir en el compromiso del empleado frente a la organización (Christian, Garza y Slaughter, 2011; Kahn 1990; Macey y Schneider, 2008).

De igual manera, diversas investigaciones han tenido como objetivo mejorar la selección del personal mediante los mejores criterios posibles (Dagdeviren y Yuksel, 2007). En esa línea, Lian y Wang (1994), por ejemplo, usan el método de matemática difusa que permite la correcta selección de personal, para lo cual utilizan variables tales como la experiencia, el nivel de compromiso, la capacidad de liderazgo y la personalidad. Mediante métodos matemáticos similares, Capaldo y Zollo (2001) plantean la lógica difusa en cuanto herramienta para la elección del candidato correcto. Karsak, Sozer y Alptekin (2003) modelan un programa de selección usando elementos cuantitativos y cualitativos. Autores como Chien y Chen (2008) identifican el uso de la minería de datos basados en árboles de decisión y reglas de asociación con el fin de plantear criterios de selección de personal. De igual manera, la literatura muestra que múltiples criterios de decisión se utilizan para la selección de personal (Bohanec et al., 1992; Gardiner y Armstrong-Wright, 2000; Jessop, 2004; Spyridakos et al., 2001; Timmermans y Vlek, 1992). En la misma línea, Dagdeviren y Yuksel (2007), así como Boran, 
Goztepe y Yavuz (2008) usan el proceso de red analítica para la selección de personal. Lin (2010) combina el proceso de red analítica y el análisis de envoltura de datos difusos para la selección de personal.

De igual forma, autores como Spencer y Spencer (1993), Rodríguez y Feliú (1996), Ansorena (1996), Woodruffe (1993) o Boyatzis (1982) definen las competencias laborales como las características subyacentes que le permiten a una persona desempeñarse en un cargo de forma correcta; por tanto, todo patrón de conducta de un individuo que le permite abordar las actividades laborales es relevante en el propósito de observar si esa persona es competente para estar en un cargo determinado.

\subsection{Satisfacción laboral y productividad}

Diversos textos muestran una relación teórica entre la satisfacción laboral, las condiciones de trabajo y la productividad laboral. En este sentido, una variable relacionada de manera significativa con la productividad laboral y la satisfacción en el trabajo es el abandono laboral. El reclutamiento y la formación de nuevo personal conlleva costos altos, además de una disminución de productividad momentánea de la empresa, ya que el nuevo empleado se adapta a sus nuevas funciones. Dado esto, la variable de abandono se ha visto correlacionada de forma negativa con la satisfacción laboral (Crampton y Wagner 1994; Hulin, Roznowski y Hachiya 1985).

Weinert (1985) identifica que el trabajo es una de las actividades que supone más tiempo en la vida, lo que genera desgaste físico y cognitivo. Autores como Lee-Ross (1993) encuentran correlaciones positivas entre la satisfacción laboral y síntomas tanto psíquicos como físicos. Del mismo modo, Jex y Gudanowski (1992), así como Schaubroeck, Ganster y Fox (1992) encuentran relaciones positivas entre la depresión y la insatisfacción laboral. Por otra parte, Palmore (1969) identifica una relación positiva entre la satisfacción laboral y la longevidad del individuo. De la misma manera, Judge y Watanabe (1993) identifican una relación positiva entre la satisfacción laboral y la satisfacción de vida. Hamermesh (1977), bajo un concepto más económico, identifica que la satisfacción está relacionada con los beneficios monetarios y no monetarios, lo que hace que un individuo sea más productivo y esté más satisfecho laboralmente, dependiendo de sus preferencias entre los factores monetarios y no monetarios.

Caldwell y O’Reilly (1990) plantean la existencia de una relación entre el mal desempeño y la insatisfacción laboral, lo cual sugiere que un empleado se desempeña bien si se siente cómodo en su trabajo. De igual forma, Bass y Barrett (1981) señalan una relación baja entre el ausentismo laboral y la satisfacción con el trabajo. Otro factor relevante en la productividad laboral es la actitud del empleado frente a la organización para la cual trabaja. Cuando el empleado tiene deseos mal intencionados contra esta puede incurrir en actos perjudiciales como, por ejemplo, robo, daños, incumplimientos de tareas, etc. De forma contraria, cuando el empleado se encuentra satisfecho con sus labores tiene más voluntad, lo que conlleva a una mayor productividad. Lo anterior muestra una relación sustancial entre el comportamiento positivo o negativo de un trabajador frente a su productividad (Mangione y Quinn, 1975). Por su parte, Gargallo-López (2008) afirma que la satisfacción laboral se encuentra directamente relacionada con el entorno, por tanto, las posibilidades de escalar en la organización y el salario es lo que motiva a los empleados a aumentar su productividad. García-Bernal, Gargallo-Castel, Marzo-Navarro y Rivera-Torres (2005) afirman que la relación con los compañeros de trabajo, su entorno laboral, además de los factores económicos, es fundamental para la satisfacción en el trabajo.

Ahora bien, desde la psicología y la gestión del talento humano Herzberg et al. (1959) plantea su teoría de los factores higiénicos y motivacionales. Los factores higiénicos hacen referencia al entorno del empleado, el cual él no puede controlar. Algunos de ellos son:

- el salario

- condiciones físicas de trabajo 
- reglamentos internos

- tipo de subordinación en el trabajo

- política fiscal de la empresa

- condiciones ambientales

Según esta teoría, la precariedad en estos factores provoca insatisfacción y el establecimiento de un punto óptimo de estos evita la insatisfacción de los empleados.

Los factores motivacionales son los que tienen una relación directa con el empleado. Algunos de ellos son:

- el desempeño laboral

- necesidades de autosuperación

- el reconocimiento laboral

- oportunidades de desarrollo

Los factores motivacionales son los que aumentan de manera sustancial la satisfacción del trabajador y el desempeño laboral. Un punto relevante en esta teoría es que cuando los factores higiénicos están satisfechos se pueden abordar los factores motivacionales.

La teoría anterior la acompaña otro planteamiento del psicólogo Maslow (1954). Este plantea la pirámide de las necesidades del hombre, las cuales se encuentran organizadas de manera jerárquica, ya que sin el cumplimiento de la más básica — la fisiológica — no se puede satisfacer la superior - la autosuperación-. De la base a la cima de la pirámide se organizan de la siguiente manera:

- Fisiológicas. Estas son las necesidades básicas, tales como respirar, comer, tener un techo, sexo, mantener la temperatura corporal, etc.

- Seguridad. Estas están relacionadas con la necesidad de estar seguro y protegido es aspectos tales como la salud, los recursos — casa, carro, dinero-, protección, etc.

- Afiliación. Estas son las necesidades que tiene un ser humano como ser social, en las cuales se incluye la amistad, la relación de pareja, la creación de familia $y$ todas las relaciones sociales en las que, por lo general, interactúa un ser humano.
- Estima. Esta necesidad se relaciona con el reconocimiento de la persona misma; en ella se incluyen variables como, por ejemplo, la autoestima, la confianza, los logros, la independencia, la reputación, el estatus, etc.

- Autorrealización. Este es el nivel más alto de la pirámide y se observa cuando la persona ha cumplido todos sus deseos en la vida y se siente una persona realizada.

Lo anterior se relaciona de forma directa con la productividad laboral, ya que si un trabajador no suple necesidades básicas como, por ejemplo, las fisiológicas, la seguridad o la afiliación, no será tan productivo como debería, ya que se sentirá desmotivado (Maslow, 1954).

\subsection{Salarios de eficiencia}

Spence (1973) establece una relación entre la productividad y la inteligencia al plantear que la inteligencia es una variable poderosa para saber qué tan productiva puede ser una persona. De igual forma, señala que el nivel de educación puede ser una señal de qué tan inteligente es un individuo. Por tanto, a una persona, entre más educada esté se le pagará más, ya que se supone que es más inteligente y, por ende, más productiva.

Por otra parte, algunos autores identifican la relación entre la productividad laboral y el salario. Desormeaux (2010) asevera que el salario de eficiencia está asociado a la productividad del trabajador. De igual forma, este comportamiento de pago por productividad, según Caraballo (1996), se encuentra asociado con el comportamiento de las empresas, ya que estas buscan maximizar sus beneficios. Dado lo anterior, autores como Romer (2002) señalan cómo si bien un salario alto genera un aumento en los costos, este también genera un aumento en la productividad y, por tanto, autores como Altman (2007) y Solow (1979) relacionan de manera positiva la productividad y el salario. 


\section{Metodología}

$\mathrm{D}$ ado que esta investigación se propone como objetivo plantear un modelo analítico que permita mejorar la elección del recurso humano en las organizaciones a fin de aumentar la eficiencia en estas, se utilizan como herramientas fundamentales de investigación la hermenéutica, la estática comparativa y la narrativa. Esto en razón a que permiten realizar análisis del marco teórico mediante la revisión de literatura, mostrar las disyuntivas de la elección del individuo y complementar el sistema complejo que se describe con la argumentación. Lo anterior basado en que Day (2005) señala cómo un artículo de revisión o reflexión busca conocer y evaluar las investigaciones sobre el tema con el fin de actualizar los conceptos de una temática en evolución. Por tanto, se utilizó el análisis de contenido, ya que Berelson (1952) identifica que es una «técnica de investigación para la descripción objetiva, sistemática y cuantitativa del contenido manifiesto de las comunicaciones con el fin de interpretarlas».

Dado lo anterior, se aborda la problemática de esta investigación de acuerdo con una búsqueda sistemática de estudios anteriores que se ven reflejados en el marco teórico, lo cual permite relacionar la importancia que tiene el nivel de educación, la preferencia por el trabajo y la preferencia por el tiempo extralaboral con el rendimiento de cada uno de los trabajadores de una organización. Luego, se plantea la estática comparativa y, por último, la narrativa del modelo a fin de complementar el sistema que se describe.

\subsection{El modelo}

De acuerdo con las investigaciones reflejadas en el marco teórico, en principio se realizará la identificación de las preferencias del individuo entre las variables cantidad de trabajo y la cantidad de actividades extralaborales. Después de identificar las preferencias de los individuos se utilizará la narrativa propuesta por Zelmer, Allen y Kesseboehmer (2006), lo que permite complementar el sistema complejo que se describe.

\subsubsection{Las variables de la estática comparativa.}

Se plantean el tiempo laboral y extralaboral como variables de elección en la estática comparativa en razón a que autores como, Hamermesh (1977), identifican que la satisfacción está relacionada con los beneficios monetarios y no monetarios; es decir, lo que hace que un individuo sea más productivo y esté más satisfecho laboralmente dependiendo de sus preferencias entre los factores monetarios y no monetarios. En la misma línea, autores como Maslow (1954) y Herzberg et al. (1959) plantean que la necesidad de afiliación y las oportunidades de desarrollo - que no necesariamente se satisfacen en la organización - son fundamentales para la satisfacción laboral y, por tanto, el aumento de la productividad que se relaciona con la eficiencia organizacional.

\subsubsection{La estática comparativa.}

Mediante el método de analogía, según Godoy (2002), se tomará una función Cobb-Douglas que se utiliza para resolver el problema de la maximización de las firmas (Cobb y Douglas, 1928), ya que esta función permite mostrar la disyuntiva de una elección. Se traza una función de bienestar del individuo $B$ que depende del tiempo dedicado al trabajo $w$ y el tiempo en actividades extralaborales $d$.

$$
B(w, d)=w^{a} d^{c}(1)
$$

Por estabilidad $a+c=1$, ya que 1 representa el $100 \%$ del tiempo.

Donde $T$ son las preferencias del individuo por las actividades laborales y actividades extralaborales, respectivamente. Con base en lo anterior, el individuo se ve restringido por el tiempo que tiene disponible, el cual se divide en las dos actividades. 


$$
\mathrm{T}(\mathrm{w}, \mathrm{d})=\mathrm{w}+\mathrm{d}(2)
$$

Donde $T$ es el tiempo total disponible, $w$ es el tiempo dedicado al trabajo y $d$ es el tiempo dedicado a actividades extralaborales.

Dadas estas ecuaciones, se plantea el método de optimización propuesto por Lagrange, Brezhneva, Tret'yakov y Wright (2012). El lagrangiano se plantea de la siguiente forma:

$$
£(w, d, \lambda)=w^{a} d^{c}-\lambda(w+d-T)
$$

Por consiguiente, se deriva parcialmente frente a .

$$
\begin{aligned}
& L^{{ }^{\prime \prime}}(w)=a w^{a-1} d^{c}-\lambda=0 \\
& L^{\prime \prime \prime}(\mathrm{d})=\mathrm{cw}^{\mathrm{a}} \mathrm{d}^{\mathrm{c}-1}-\lambda=0 \\
& L^{\prime \prime \prime}(\lambda)=w+d-T=0
\end{aligned}
$$

$\mathrm{Al}$ resolver el sistema de ecuaciones se encuentra que:

$$
\begin{aligned}
& w=\frac{a T}{(a+c)}(3) \\
& w=\frac{c T}{(a+c)}(4)
\end{aligned}
$$

Dado que, entonces:

$$
\begin{aligned}
& w^{*}=a T(5) \\
& d^{*}=c T(6)
\end{aligned}
$$

Lo anterior permite identificar que la cantidad de tiempo que se le dedica a las actividades laborales y extralaborales depende de las preferencias del individuo por cada una multiplicadas por el tiempo total.

De igual forma la función de bienestar óptima está dada por:

$$
\mathrm{B}^{*}(\mathrm{a}, \mathrm{c})=\mathrm{a}^{\mathrm{a}} \mathrm{c}^{\mathrm{c}} \mathrm{T}(7)
$$

Por otra parte, la sustituibilidad del tiempo de trabajo respecto al tiempo extralaboral está dada por:

$$
\frac{B(w)^{\prime}}{B(d)^{\prime}}=\frac{a w^{a-1} d^{c}}{c w^{a} d^{c-1}}=\frac{a d}{c w}
$$

$\mathrm{Al}$ remplazar (5) y (6) en (8):

$$
\frac{B(w)^{\prime}}{B(d)^{\prime}}=(1)
$$

Esto quiere decir que a fin de obtener una unidad más de tiempo de trabajo, el individuo debe renunciar a una unidad del tiempo extralaboral.

El modelo permite identificar que la satisfacción del individuo depende de la disponibilidad de tiempo que está dispuesto a permanecer en el trabajo y del tiempo que está dispuesto a destinar a realizar actividades extralaborales. Por otra parte, Spence (1973) identifica que la productividad del individuo está asociada a la inteligencia, pero dado que medir la inteligencia tiene algunas dificultades, el nivel de educación permitiría acercarse a la capacidad intelectual que tiene (Mincer, 1991).

\subsubsection{Narrativa del modelo.}

Se puede identificar que dos variables fundamentales para el buen desempeño en un cargo son: el tiempo que el individuo está dispuesto a permanecer trabajando y el nivel de educación que este tiene. En este sentido, la figura 1 propone el grado de educación y el grado de sacrificio de tiempo que debe tener una persona en los diferentes cargos de la empresa. Para esto se supondrá una empresa industrial en la que los cargos inferiores son los de oficios varios como, por ejemplo, el de vigilancia y aseo. En el caso de los servicios de vigilancia el individuo debe tener bajo nivel educativo y, a su vez, gran disponibilidad de sacrificio de tiempo (Espitia, 2010), ya que una función como esta requiere de la disponibilidad de la persona en las noches y los fines de semana. Lo anterior quiere decir que este individuo, aunque tenga bajo nivel de educación, compensa sus ingresos sacrificando gran parte de su tiempo de vida. En los cargos de aseadores, por ejemplo, existe una mayor tendencia a ser ocupados por mujeres. Esto se puede deber a que, a diferencia del trabajo de vigilancia, este necesita una menor disponibilidad de tiempo, ya que por factores fisiológicos y sociales la mujer ocupa un mayor tiempo en actividades extralaborales, como lo son los oficios de la casa y la crianza de los niños. 
A fin de analizar los puestos intermedios de una organización supondremos a un gerente de ventas y un gerente de mercadeo. La función esencial de un gerente de ventas es la consecución de los ingresos de la empresa por medio de la actividad comercial (Vásquez, 2008). Dado que la naturaleza de una organización es maximizar los beneficios, este gerente debe estar dispuesto a la consecución constante de estos. En razón a lo anterior, la consecución constante de ingresos se encuentra relacionada con la búsqueda constante de clientes, lo cual requiere de un mayor desgaste físico que intelectual. Por otra parte, un gerente de mercadeo es el encargado de realizar la estrategia que adoptará la organización para la venta del producto (Contreras y Ramos, 2016), lo que implica un alto conocimiento de variables de mercado tales como tendencias, precios y la situación actual de la economía, lo que genera un mayor desgaste intelectual que físico.

Dado que el gerente de mercadeo y el de ventas son de igual relevancia, pero tienen características diferentes - pues uno hace un mayor desgaste físico y otro intelectual- el desgaste físico del gerente de ventas debe ser equivalente al desgaste intelectual del gerente de mercadeo para cumplir la teoría de salarios de eficiencia que plantea Desormeaux (2010). La importancia de la teoría de salarios de eficiencia viene dada por la productividad marginal del individuo, lo que permite inferir que la productividad por hora del gerente de mercadeo debe ser mayor que la del gerente de ventas, puesto que el gerente de ventas tiene una actividad de mayor desgaste de tiempo, pero los dos tienen impactos similares en resultados.

Dadas las circunstancias en las cuales se ven involucrados estos dos tipos de gerentes, en la elección de un gerente de ventas debe prevalecer, sobre todo, el tiempo que el individuo está dispuesto a trabajar más que su nivel intelectual. Por otra parte, en la elección de un gerente de mercadeo debe prevalecer en mayor medida la capacidad intelectual con respecto al tiempo que está dispuesto a dedicarle al trabajo. Como se ha mencionado, identificar el nivel intelectual de un individuo es supremamente difícil, de modo que un proxy de este es el nivel de estudios (Mincer, 1991). Por esta razón, un gerente de mercadeo debe tener un nivel de estudios mayor que un gerente de ventas. Cabe resaltar que los salarios de los dos individuos deben ser iguales, ya que, si bien ser gerente de ventas desgasta más tiempo, el impacto del gerente de mercadeo es similar por el desgaste intelectual.

En el caso de la alta gerencia se debe tener claro que la persona que la ocupe debe ser un individuo con alto nivel de conocimiento y competencias (Alles, 2008). De igual forma, debe estar dispuesto a tener un alto desgaste de tiempo, lo que nos permite inferir que la cabeza de una organización ha de ser un gran ejecutivo con altos niveles de estudios. En el caso de la organización más compleja que puede existir en un país, esto es, el Estado, el presidente de una nación debe ser un gran ejecutivo con altos niveles de conocimiento, de tal forma que tome las mejores decisiones para la sociedad.

Este modelo puede dar luces sobre la identificación de las diferencias salariales entre hombres y mujeres, ya que diferentes estudios identifican que mujeres con cargos y un nivel de estudios similares obtienen salarios menores con relación a los de los hombres. Este modelo permite inferir que la disponibilidad de tiempo de las mujeres influye en su remuneración salarial, y en algunos casos la ausencia de las mujeres en algunos cargos. Esta problemática deben abordarla el Estado y las organizaciones a fin de tratar de generar una nivelación salarial. 
Figura 1. Nivel de sacrificio de tiempo vs. nivel de educación

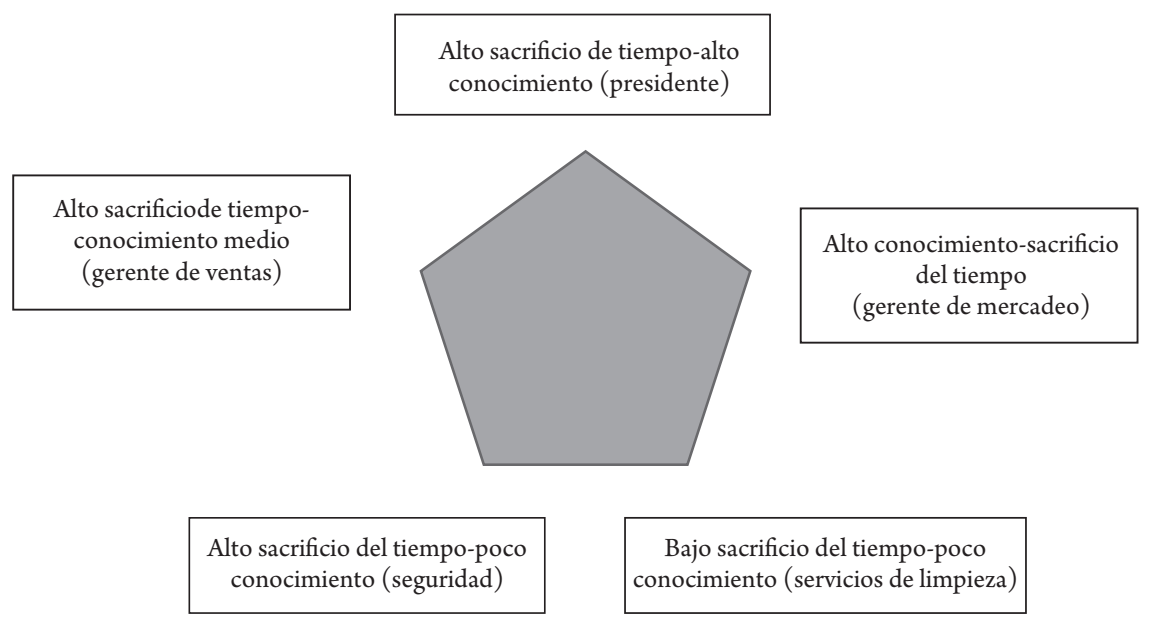

Fuente. Elaboración propia.

\section{Conclusiones}

$\mathrm{E}$ ste documento tuvo como objetivo plantear un modelo analítico que permita mejorar la elección del recurso humano en las organizaciones a fin de aumentar la eficiencia en las organizaciones. Para esto se usaron como herramientas fundamentales de investigación la hermenéutica, la estática comparativa y la narrativa, con la finalidad de realizar un modelo que contiene análisis de contenido, modelación matemática y la argumentación para describir el sistema complejo que se aborda. Al realizar la revisión de literatura y contemplar algunas de las variables relevantes en el momento de reclutar una persona y aumentar las posibilidades de que el individuo se desempeñe de manera correcta en el cargo, se utilizó el método de analogía (Godoy, 2002), tomando una función Cobb-Douglas que se utilizó para resolver el problema de maximización de las firmas planteada por Cobb y Douglas (1928) y la narrativa propuesta por Zelmer, Allen y Kesseboehmer (2006).

El modelo planteado se basa en tres variables fundamentales: el tiempo que el individuo está dispuesto a permanecer trabajando, el tiempo extralaboral del individuo y el nivel de educación que tiene. El modelo tiene como primicia inicial que las personas cuentan con dos espacios de tiempo - el tiempo laboral y el extralaboral - en los que el individuo, de acuerdo con sus preferencias, decide seleccionar la cantidad adecuada de los dos espacios temporales, ya que para adquirir una unidad más de uno debe renunciar a una unidad del otro. Esta elección del tiempo es relevante para la selección del personal, ya que existen diversos cargos con dedicación temporal distinta.

Por otra parte, la tercera variable contemplada en el modelo es el nivel de educación, ya que cada uno de los cargos ejecutados en la empresa necesitan personas con diferentes niveles formativos. La identificación de esta variable se basa en los planteamientos de Spence (1973), ya que este identifica cómo la productividad del individuo está asociada a la inteligencia, pero dado que medir la inteligencia tiene algunas dificultades el nivel de educación permitiría acercarse a la capacidad intelectual que tiene la persona (Mincer, 1991). En el caso de la alta gerencia, la persona que ocupe dicho cargo debe ser un individuo con alto nivel de conocimiento que permita reducir la incertidumbre a la hora de tomar decisiones, así como que esté 
dispuesto a sacrificar mucho de su tiempo trabajando. Por otra parte, el servicio de vigilancia lo debe ocupar una persona que esté dispuesta a sacrificar mucho de su tiempo en el trabajo, pero que tenga un nivel bajo de educación.

Dado lo anterior, es posible identificar que dos variables fundamentales para el buen desempeño

\section{Referencias}

Alles, M. (2008). Comportamiento organizacional. Argentina: Ediciones Granica. DOI: https://doi.org/10.2139/ ssrn. 1152105

Altman, M. (2007). Effort discretion and economic agency and behavioral economics: transforming economic theory and public policy. En F. Roger (Ed.) Renaissance in behavioral economics Harvey Leibenstein's impact of contemporary economic analysis. (119-159). Nueva York: Routledge.

Ansorena, A. de. (1996). 15 pasos para la selección de personal con éxito. Barcelona. Editorial Paidos.

Bass, B. M.; Barrett, G. V. (1981). People, work, and organizations: an introduction to industrial and organizational psychology. Boston: Allyn \& Bacon.

Berelson, B. (1952). Content analysis in communication research. Nueva York: The Free Press.

Brezhneva, O.; Tret'yakov, A. A.; Wright, S. E. (2012). A short elementary proof of the Lagrange multiplier theorem. Optimization Letters, 6(8), 1597-1601. DOI: https://doi. org/10.1007/s11590-011-0349-4

Bohanec, M.; Urh, B.; Rajkovič, V. (1992). Evaluating options by combined qualitative and quantitative methods. Acta Psychologica, (80), 67-89. DOI: https://doi. org/10.1016/0001-6918(92)90041-B

Boran, S.; Goztepe, K.; Yavuz, E. (2008). A study on election of personnel based on performance measurement by using analytic network process (ANP). International Journal of Computer Science and Network Security, 8(4), 333- 340. Recuperado de https://bit.ly/36OwzJm

Boyatzis, R. E. (1982). The competent manager: a model for effective performance. Nueva York: John Wiley \& Sons.

Caldwell, D. F.; O'Reilly III, C. A. (1990). Measuring personjob fit with a profile-comparison process. Journal of Applied Psychology, 75(6), 648-657. DOI: https://doi. org/10.1037/0021-9010.75.6.648 en un cargo son el tiempo que el individuo está dispuesto permanecer trabajando y el nivel de educación que tiene. Por último, se debe tener en cuenta que este modelo no contempla variables tales como la experiencia, el tamaño de la organización y la capacidad de relacionarse de los individuos, las cuales son puntos relevantes para algunos cargos.

Caraballo, P. (1996). Salarios, productividad y empleo: la hipótesis de los salarios de eficiencia. Revista Cuadernos de Estudios Empresariales, 6, 105-127. Recuperado de https://bit. ly/2XntdK7

Capaldo, G.; Zollo, G. (2001). Applying fuzzy logic to personnel assessment: a case study. Omega, 29(6), 585-597. DOI: https://doi.org/10.1016/S0305-0483(01)00047-0

Chien, C.F.; Chen, L.F. (2008). Data mining to improve personnel selection and enhance human capital: a case study in hightechnology industry. Expert Systems with Applications, 34(1), 280-290. DOI: https://doi.org/10.1016/j.eswa.2006.09.003

Christian, M. S.; Garza, A. S.; Slaughter, J. E. (2011). Work engagement: a quantitative review and test of its relations with task and contextual performance. Personnel Psychology, 64(1), 89-136. DOI: https://doi.org/10.1111/j.17446570.2010.01203.x

Cobb, C.W.; P.H. Douglas (1928). A theory of production. American Economic Review, 18(1), 139-165. Recuperado de www.jstor.org/stable/1811556

Collings, D. G.; Mellahi, K. (2009). Strategic talent management: a review and research agenda. Human Resource Management Review, 19(4), 304-313. DOI: https://doi.org/10.1016/j. hrmr.2009.04.001

Contreras, F. L.; Ramos, M. L. Z. (2016). What is marketing? A study on marketing managers' perception of the definition of marketing. Forum Empresarial, 21(1), 49-69. DOI: https:// doi.org/10.33801/fe.v21i1.4087

Crampton, S. M.; Wagner III, J. A. (1994). Percept-percept inflation in microorganizational research: an investigation of prevalence and effect. Journal of Applied Psychology, 79(1), 6776. DOI: https://doi.org/10.1037/0021-9010.79.1.67

Dagdeviren, M. (2010). A hybrid multi-criteria decision-making model for personnel selection in manufacturing systems. Journal of Intelligent Manufacturing, 21(4), 451-460. DOI: https://doi.org/10.1007/s10845-008-0200-7 
Dagdeviren, M.; Yuksel, I. (2007). Personnel selection using analytic network process. Istanbul Ticaret Universitesi Fen Bilimleri Dergisi Yil, 6(11), 99-118. Recuperado de https:// bit.ly/3cpaIJN

Day, R. A. (2005). Cómo escribir y publicar trabajos científicos. Washington: Organización Panamericana de la Salud. Publicación Científica y Técnica 598.

Desormeaux, N. (19 de mayo de 2010). Salarios de eficiencia y productividad Valparaíso, Chile: Universidad Técnica Federico Santa María.

Espitia, Y. P. (2010). La selección por competencias aplicada en la seguridad privada (Trabajo de grado). Universidad Militar Nueva Granada, Bogotá, Colombia. Recuperado de https:// bit.ly/36PiXNO

García-Bernal, J.; Gargallo-Castel, A.; Marzo-Navarro, M.; RiveraTorres, P. (2005). Job satisfaction: empirical evidence of gender differences. Women in Management Review, 20(4), 279288. DOI: https://doi.org/10.1108/09649420510599098

Gardiner, A. R.; Armstrong-Wright, D. (2000). Employee selection and anti- discrimination law: implications for multi-criteria group decision support. Journal of MultiCriteria Decision Analysis, 9, 99-109. DOI: https://doi. org/10.1002/1099-1360(200001/05)9:1/3\%3C99::AIDMCDA271\%3E3.0.CO;2-G

Gargallo-López, B. (2008). Estilos de docencia y evaluación de los profesores universitarios y su influencia sobre los modos de aprender de sus estudiantes. Revista Española de Pedagogía, LXVI(241), 425-446. Recuperado de https://bit.ly/2Xqg970

Guest, D. E. (2014). Employee engagement: a skeptical analysis. Journal of Organizational Effectiveness: People and Performance, 1(2), 141-156. DOI: https://doi.org/10.1108/ JOEPP-04-2014-0017

Godoy, L. A. (2002). Sobre la estructura de las analogías en ciencias. Interciencia, 27(8), 422-429. Recuperado de https:// bit.ly/2TZVSDc

Hamermesh, D. S. (1977). Economic aspects of job satisfaction. En O. Ashenfelter; W. Oates (Ed.) Essays in Labor Market Analysis. (53-72). Nueva York: John Wiley.

Harter, J. K.; Blacksmith, N. (2010). Employee engagement and the psychology of joining, staying in, and leaving organizations. En P. A. Linley; S. Harrington; N. Garcea (Eds.) Oxford handbook of positive psychology and work. (121130). Oxford: Oxford. DOI: https://doi.org/10.1093/ oxfordhb/9780195335446.013.0010

Herriot, P. (2002). Selection and self: selection as a social process. European Journal of Work and Organizational Psychology, 11(4), 385-402. DOI: https://doi. org/10.1080/13594320244000256

Herzberg, F.; Mausner, B.; Snyderman, B. (1959). The motivation to work. Nueva York: John Wiley \& Sons.
Hulin, C. L.; Roznowski, M.; Hachiya, D. (1985). Alternative opportunities and withdrawal decisions: empirical and theoretical discrepancies and an integration. Psychological Bulletin, 97(2), 233-250. DOI: https://doi. org/10.1037/0033-2909.97.2.233

Jessop, A. (2004). Minimally biased weight determination in personnel selection. European Journal of Operational Research, 153(2), 433-444. DOI: https://doi.org/10.1016/S03772217(03)00163-2

Jex, S. M.; Gudanowski, D. M. (1992). Efficacy beliefs and work stress: an exploratory study. Journal of Organizational Behavior, 13(5), 509-517. DOI: https://doi.org/10.1002/ job.4030130506

Judge, T. A.; Watanabe, S. (1993). Another look at the job satisfaction-life satisfaction relationship. Journal of Applied Psychology, 78(6), 939-948. DOI: https://doi. org/10.1037/0021-9010.78.6.939

Kahn, W. A. (1990). Psychological conditions of personal engagement and disengagement at work. Academy of Management Journal, 33(4), 692-724. DOI: https://doi. org $/ 10.5465 / 256287$

Karsak, E. E.; Sozer, S.; Alptekin, S. E. (2003). Production planning in quality function deployment using a combined analytical network process and goal programming approach. Computers and Industrial Engineering, 44(1), 171-190. DOI: https://doi.org/10.1016/S0360-8352(02)00191-2

Kristof, A. L. (2006). Person-organization fit: an integrative review of its conceptualizations, measurement, and implications. Personnel Psychology, 49(1), 1-49. DOI: https:// doi.org/10.1111/j.1744-6570.1996.tb01790.x

Lee-Ross, D. (1993). Two styles of management, two types of worker. International Journal of Contemporary Hospitality Management, 5(4), 20-24. DOI: https://doi. org/10.1108/09596119310042073

Liang, S. L.; Wang, M. J. (1994). Personnel selection using Fuzzy MCDM Algorithm. European Journal of Operational Research, 78(1), 22-33. DOI: https://doi.org/10.1016/03772217(94)90119-8

Lin, H. C. (2010). Personnel selection using analytic network process and fuzzy data envelopment analysis approaches. Computers \& Industrial Engineering, 59(4), 937-944. DOI: https://doi.org/10.1016/j.cie.2010.09.004

Macey, W. H.; Schneider, B. (2008). The meaning of employee engagement. Industrial and Organizational Psychology: Perspectives on Science and Practice, (1), 3-30. DOI: https:// doi.org/10.1111/j.1754-9434.2007.0002.x

Mangione, T. W.; Quinn, R. P. (1975). Job satisfaction, counterproductive behavior, and drug use at work. Journal of Applied Psychology, 60(1), 114-116. DOI: https://doi. org $/ 10.1037 / \mathrm{h} 0076355$ 
Maslow, A. H. (1954). Motivación y personalidad. Nueva York: Harper \& Row.

May, D. R.; Gilson, R. L.; Harter, L. M. (2004). The psychological conditions of meaningfulness, safety and availability and the engagement of the human spirit at work. Journal of Occupational and Organizational Psychology, 77, 11-37. DOI: https://doi.org/10.1348/096317904322915892

Mayo, E. (1933). The human problems of an industrial civilization. Salem, NH: Ayer.

Mincer, J. (Septiembre de 1991). Education and unemployment (Working paper No. w3838). Cambridge, National Bureau of Economic Research. DOI: https://doi.org/10.3386/w3838

Morgeson, F. P.; Dierdorff, E. C. (2011). Work analysis: from technique to theory. En S. Zedeck (Ed.), APA Handbook of Industrial and Organizational Psychology. (Vol. 2, 3-41). Washington, DC: American Psychological Association. DOI: https://doi.org/10.1037/12170-001

Palmore, E. (1969). Predicting longevity: a follow-up controlling for age. The Gerontologist, 9(4), 247-250. DOI: https://doi. org/10.1093/geront/9.4_Part_1.247

Rodríguez, N.; Feliú, P. (1996). Curso básico de psicometría. Caracas: Escuela de Psicología, Universidad Central de Venezuela.

Romer, D. (2002). Macroeconomía avanzada. Madrid: McGrawHill.

Saks, A. M.; Gruman, J. A. (2011). Getting newcomers engaged: the role of socialization tactics. Journal of Managerial Psychology, 26(5), 383-402. DOI: https://doi. org/10.1108/02683941111139001

Schaubroeck, J.; Ganster, D. C.; Fox, M. L. (1992). Dispositional affect and work-related stress. Journal of Applied Psychology, 77(3), 322-335. DOI: https://doi.org/10.1037/00219010.77.3.322

Shafritz, J. M.; Ott, J. S.; Jang, Y. S. (2015) Classics of organization theory. UK: Cengage Learning.

Solow, R. M. (1979). Another possible source of wage stickiness. Journal of Macroeconomics, 1(1), 79-82. DOI: https://doi. org/10.1016/0164-0704(79)90022-3
Spence, M. (1973). Market signalling: information transfer in hiring and related processes. Cambridge: Harvard University Press.

Spencer, L. M.; Spencer, S. M. (1993). Competence at work. Nueva York: Jhon Wiley \& Sons.

Spyridakos, A.; Siskos, Y.; Yannacopoulos, D.; Skouris, A. (2001). Multicriteria job evaluation for large organizations. European Journal of Operational Research, 130(2), 375-387. DOI: https://doi.org/10.1016/S0377-2217(00)00039-4

Taylor, F. W. (1914). Scientific management: reply from Mr. FW Taylor. The Sociological Review, 7(3), 266-269. DOI: https:// doi.org/10.1111/j.1467-954X.1914.tb02387.x

Timmermans, D.; Vlek, C. (1992). Multi-attribute decision support and complexity: an evaluation and process analysis of aided versus unaided decision making. Acta Psychologica, 80(1-3), 49-65. DOI: https://doi.org/10.1016/00016918(92)90040-K

Vance, R. J. (2006). Employee engagement and commitment: a guide to understanding, measuring, and increasing engagement in your organization; effective practice guidelines. Alexandria, VA: Society for Human Resource Management.

Verquer, M. T.; Beehr, T. A.; Wagner, S. H. (2003). A metaanalysis of the relationship between person-organization fit and work-related attitudes. Journal of Vocational Behavior, 63(3), 473-489. DOI: https://doi.org/10.1016/S00018791(02)00036-2

Vásquez, G. (2008). Las ventas en el contexto gerencial latinoamericano. Temas de Management, 6(2), 16-22. Recuperado de https://bit.ly/2MlyETy

Weinert, A. B. (1985). Manual de psicología de la organización: la conducta humana en las organizaciones. Barcelona: Herder.

Woodruffe, C. (1993). What is meant by a competency? Leadership \& Organization Development Journal, 14(1), 29-36. https://doi.org/10.1108/eb053651

Zellmer, A. J.; Allen, T. F.; Kesseboehmer, K. (2006). The nature of ecological complexity: a protocol for building the narrative. Ecological Complexity, 3(3), 171-182. DOI: https://doi. org/10.1016/j.ecocom.2006.06.002 